\title{
Ligand-Induced Surface Charge Density Modulation Generates Local Type-II Band Alignment in Reduced-Dimensional Perovskites
}

Rafael Quintero-Bermudez ${ }^{1 \ddagger}$, Andrew H. Proppe ${ }^{1,2 \ddagger}$, Arup Mahata ${ }^{3,4 \ddagger}$, Petar Todorovic ${ }^{1}$, Shana O. Kelley ${ }^{2,5}$, Filippo De Angelis ${ }^{3,4,6 *}$, Edward H. Sargent ${ }^{1 *}$

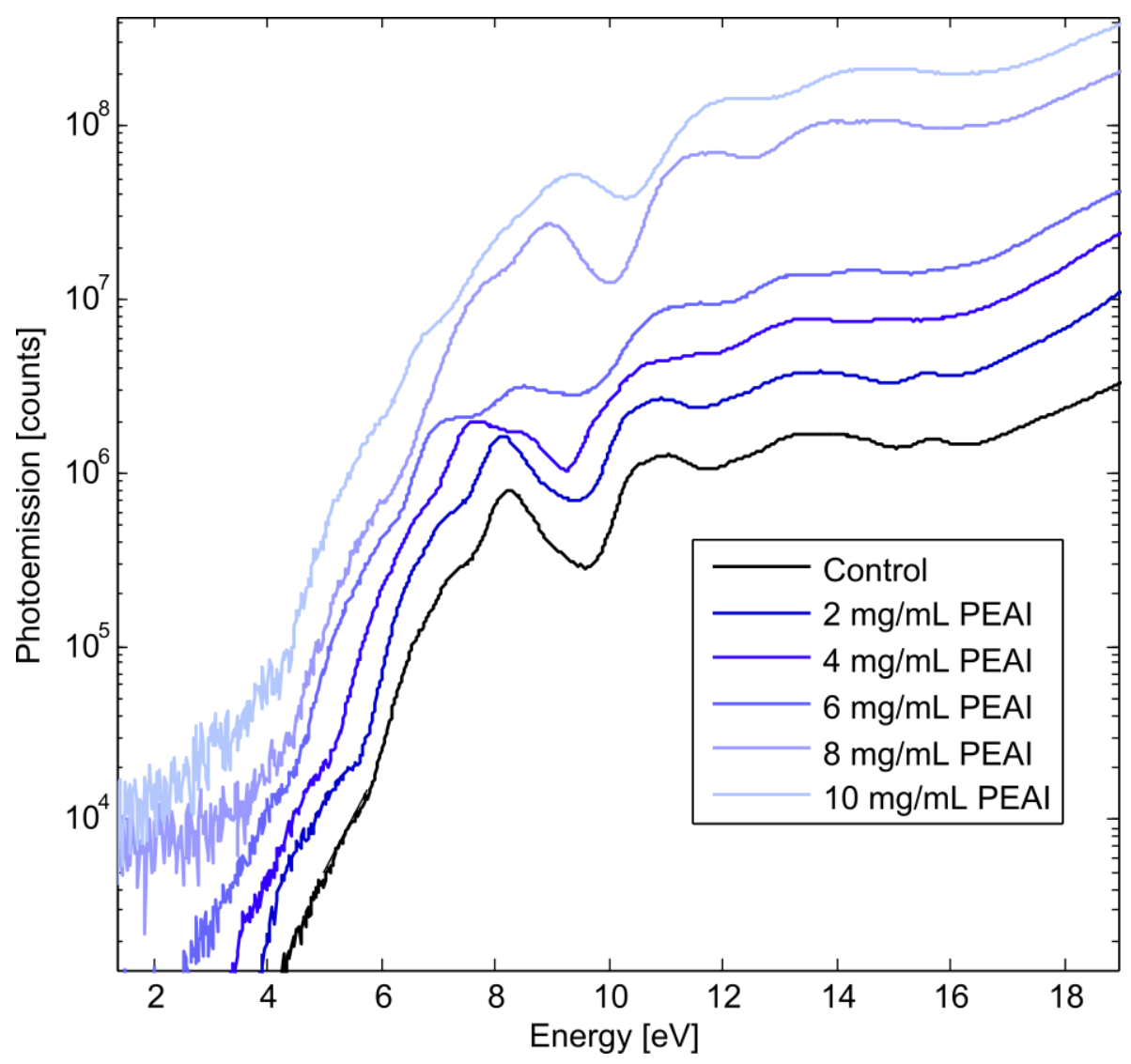

Figure S1. UPS data for increasing PEAI concentration in treatments. As treatment concentration is increased beyond $6 \mathrm{mg} / \mathrm{mL}$ it becomes hard to determine the photoemission onset due to blurring/merging of features. In addition, the appearance of multiple peaks makes it hard to discern peaks within the valence spectra. 


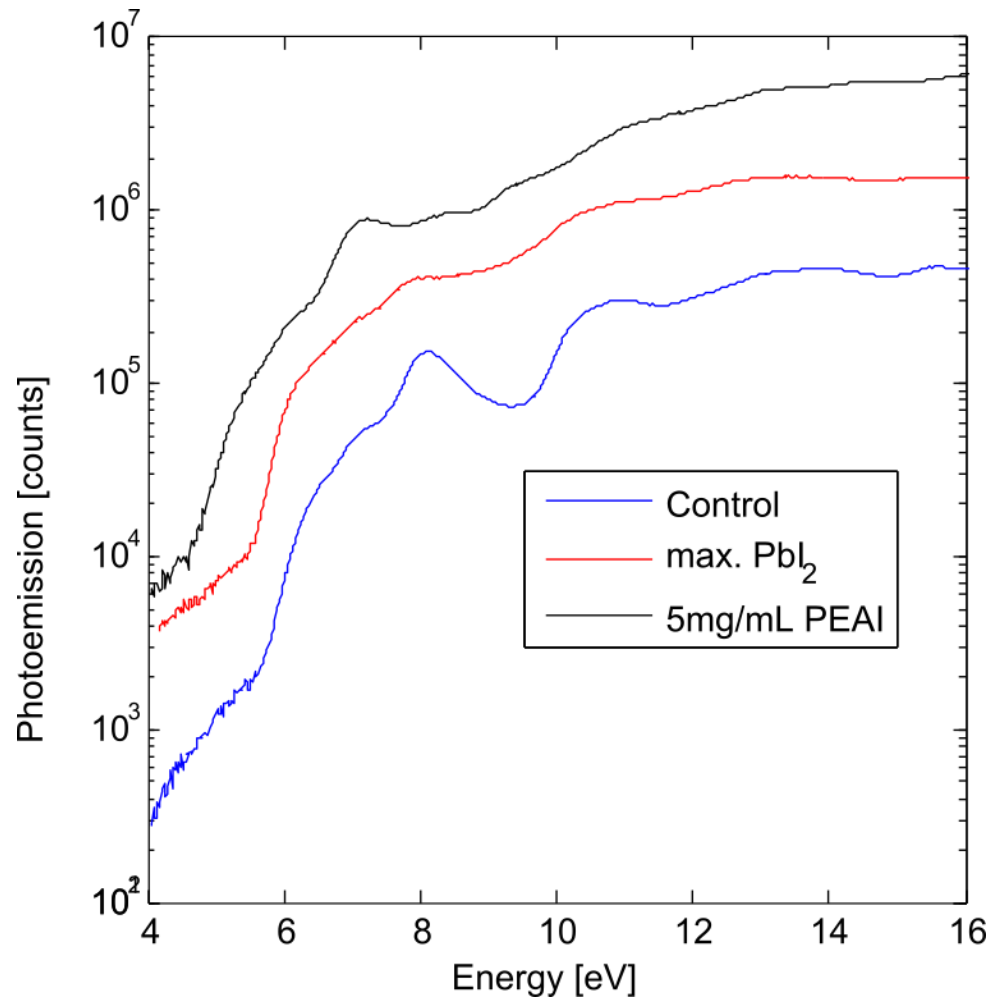

Figure S2. UPS data for $\mathbf{P b I}_{2}$ and PEAI treatments. It is evident that while $\mathrm{PbI}_{2}$ treatments to not alter the work function, PEAI treatments clearly shift the positioning of bands.

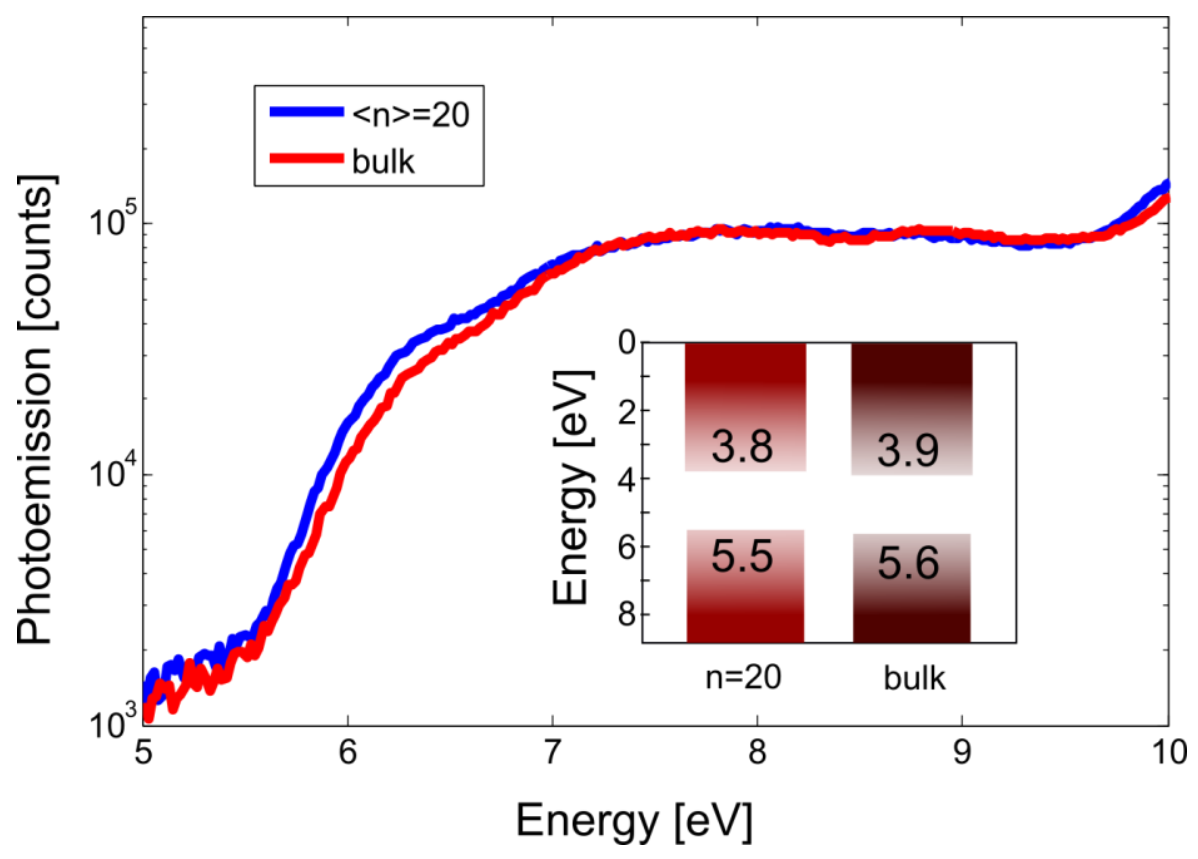

Figure S3. UPS data for $<\mathbf{n}>=\mathbf{2 0}$ and bulk films. The bands are shifted up in the RDP film due to the prevalence of PEAI surfaces relative to the bulk perovskite film. 


\section{PEA-coverage Work Function}

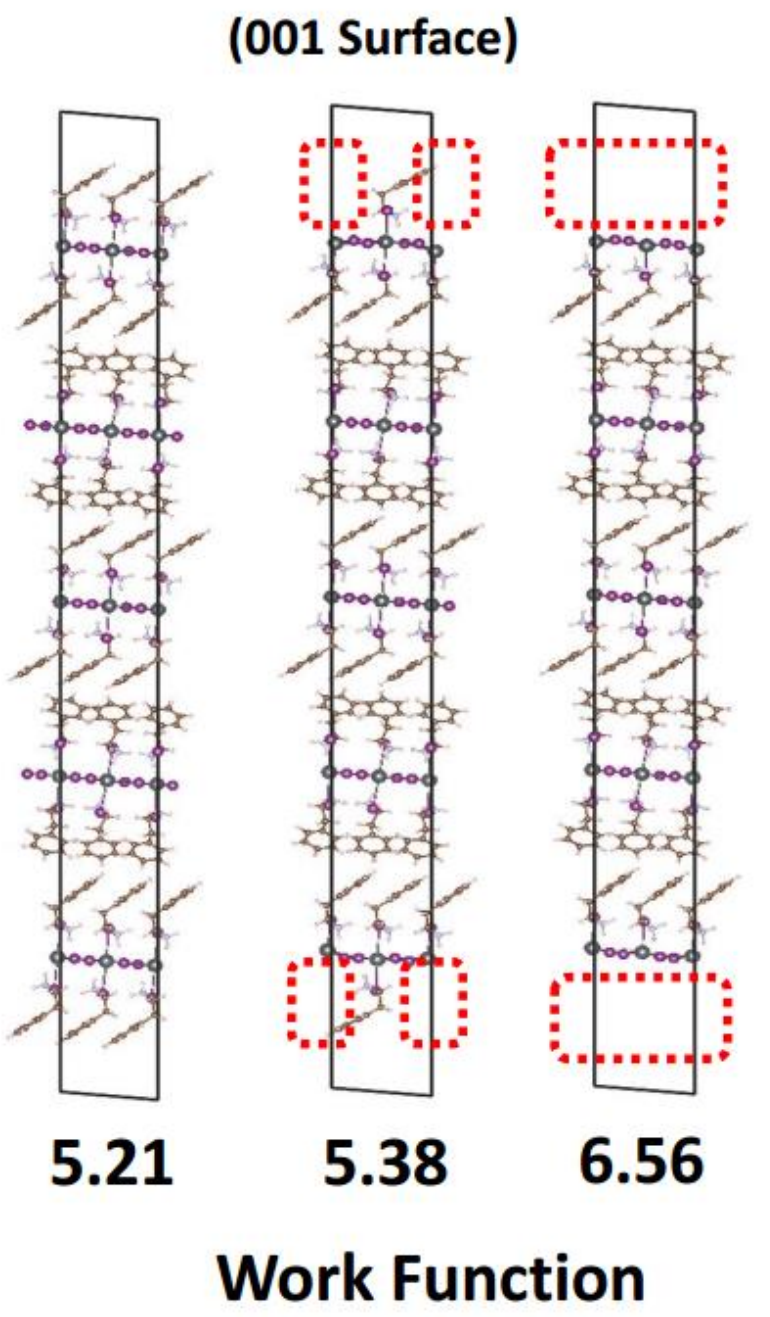

(100 Surface)

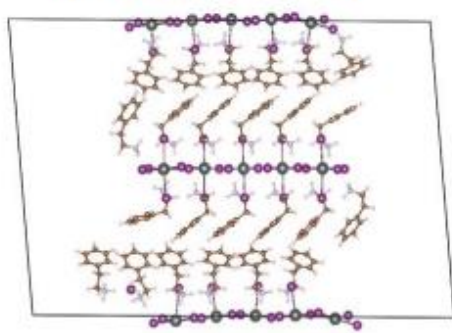

4.99

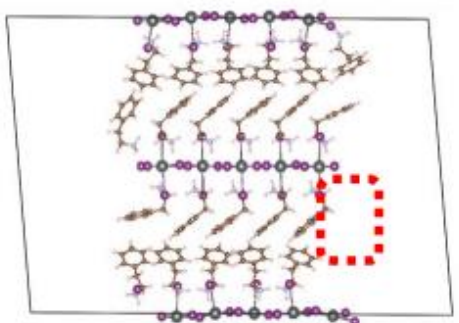

5.81

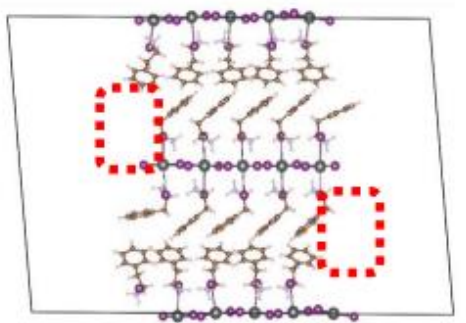

6.37

Figure S4. Work Function of PEAI treatments on PEA-based $n=1$ perovskite. Despite minor differences in the exact work function after PEAI additions at different surfaces, there is a clear trend for the reduction in work function as the amount of PEAI is increased. 


\section{BTA-coverage Work Function}

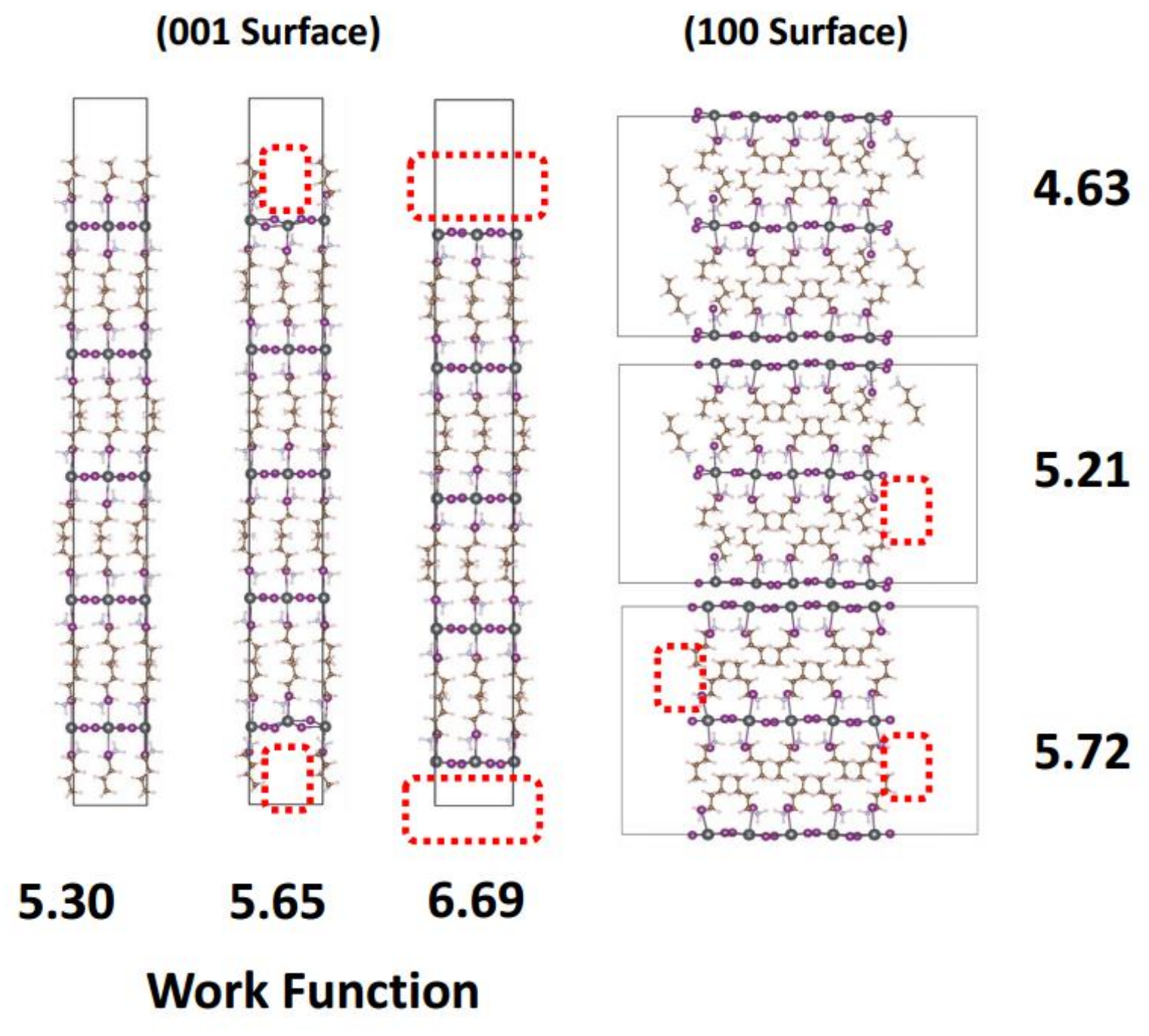

Figure S5. Work Function of BTAI treatments on BTA-based n=1 perovskite. Despite minor differences in the exact work function after BTAI additions at different surfaces, there is a clear trend for the reduction in work function as the amount of PEAI is increased. 


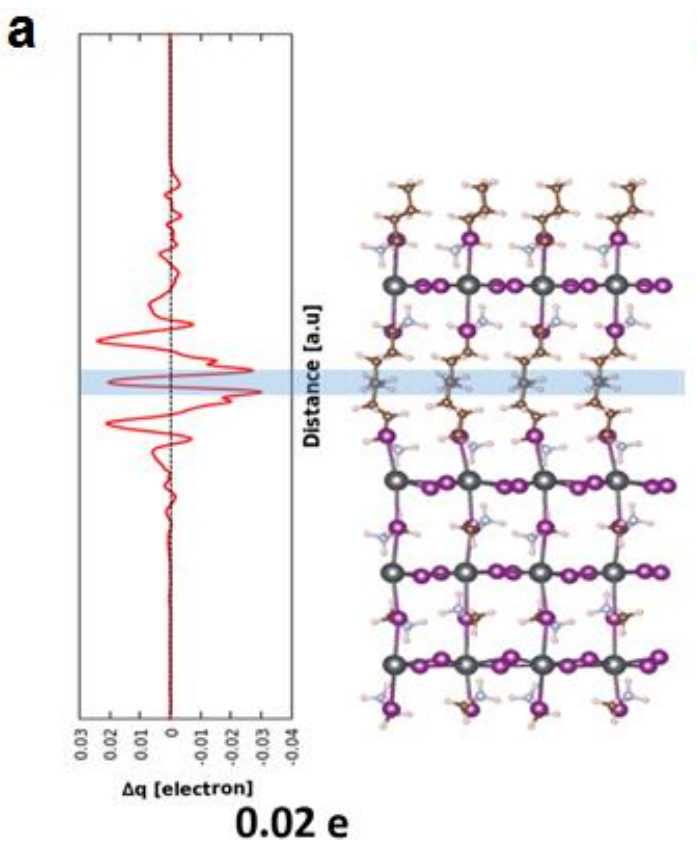

BAl-ter

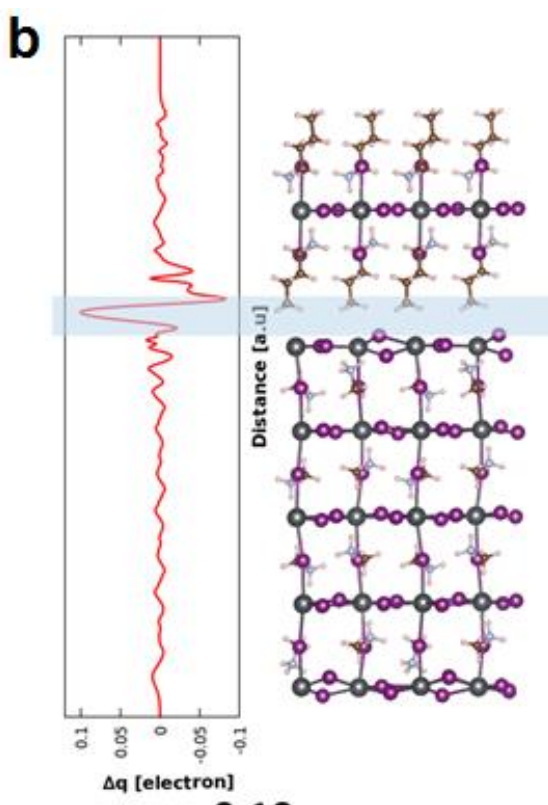

0.10 e

$\mathrm{Pbl}_{2}$-ter

Figure S6. Calculated charge transfer across an RDP interface with different surface termination. Calculated for (a) BAI-terminated and (b) PbI2-terminated structures.

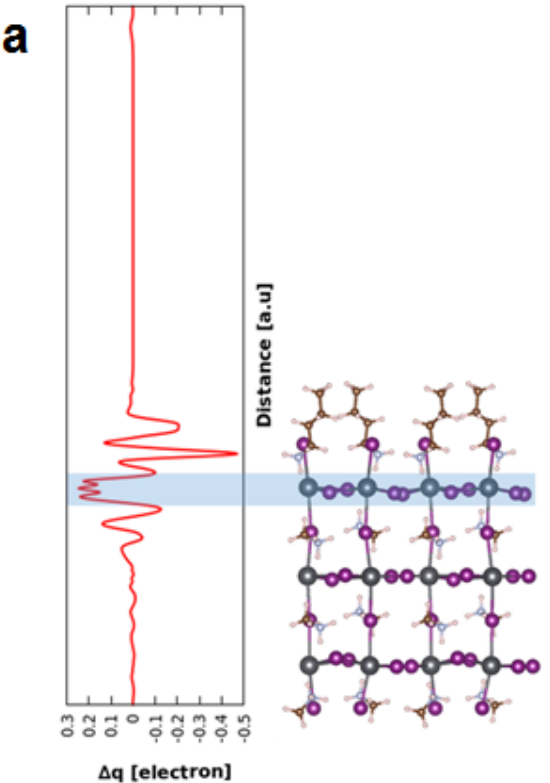

0.25 e

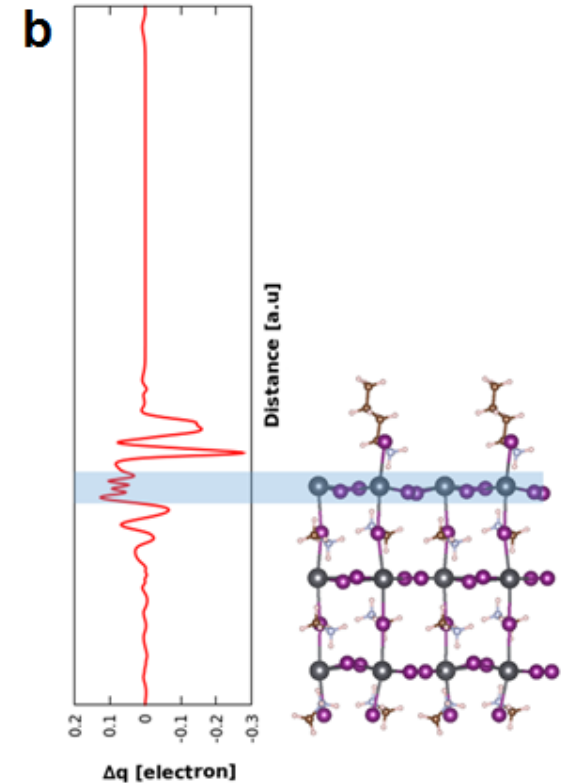

0.13 e

Figure S7. Calculated charge transfer with varying BAI-concentration at the interface. We have further quantified charge transfer with the varying BAI coverage by varying the density of 
BAI-units on a BAI-terminated $n=3$ perovskite surface. This reveals a linear decrease of charge transfer contribution with decreasing BAI-units. It should be expected that the dipole moment would increase as the density of dipole-containing molecules (BAI, in this case) is increased. This set of calculations confirms that surface charge density redistribution also increases as a function of ligand density.
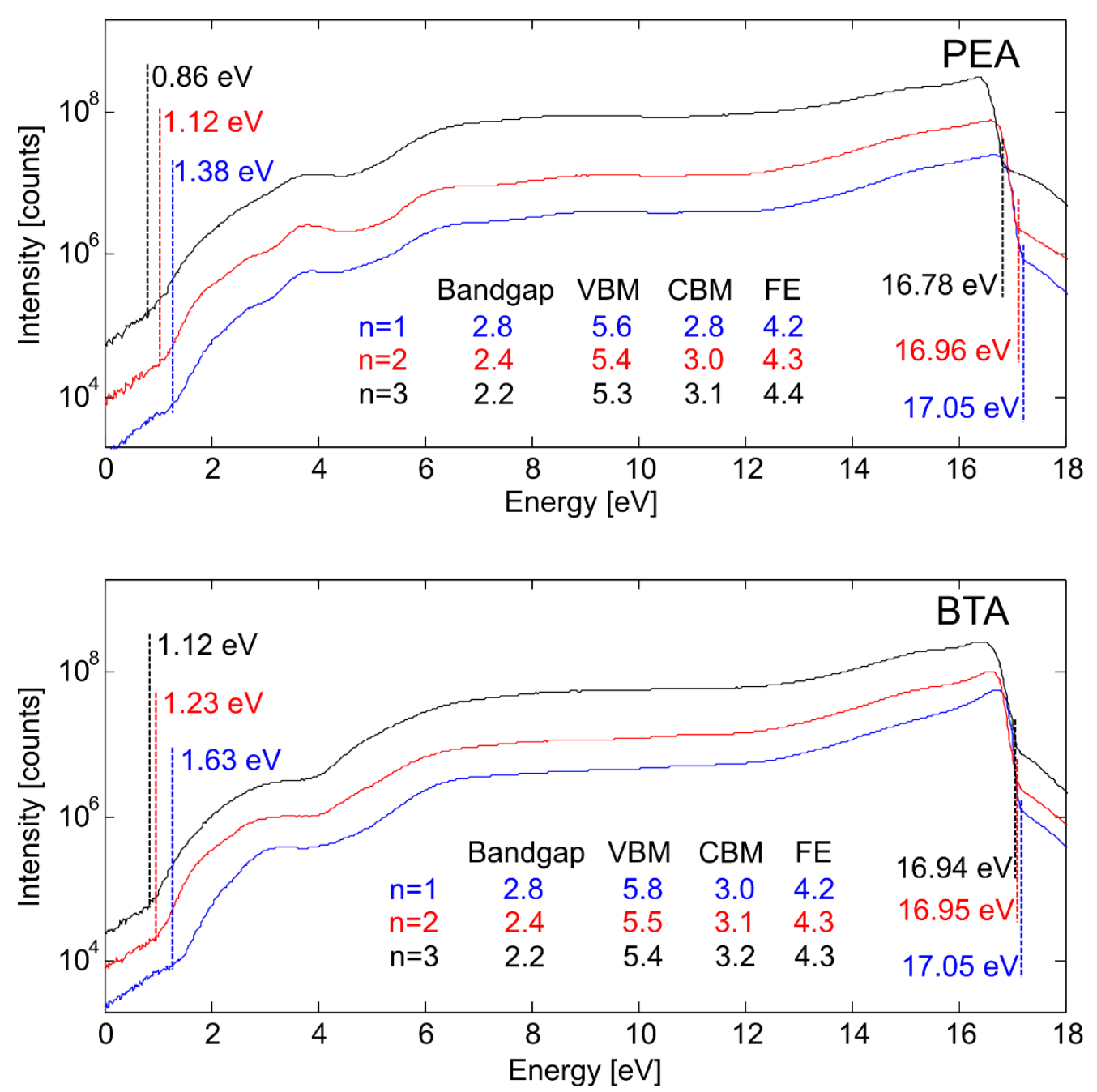

Figure S8. Full Ultraviolet Photoelectron Spectroscopy raw data. Data is presented after bias correcting (which was $5 \mathrm{~V}$ ). 

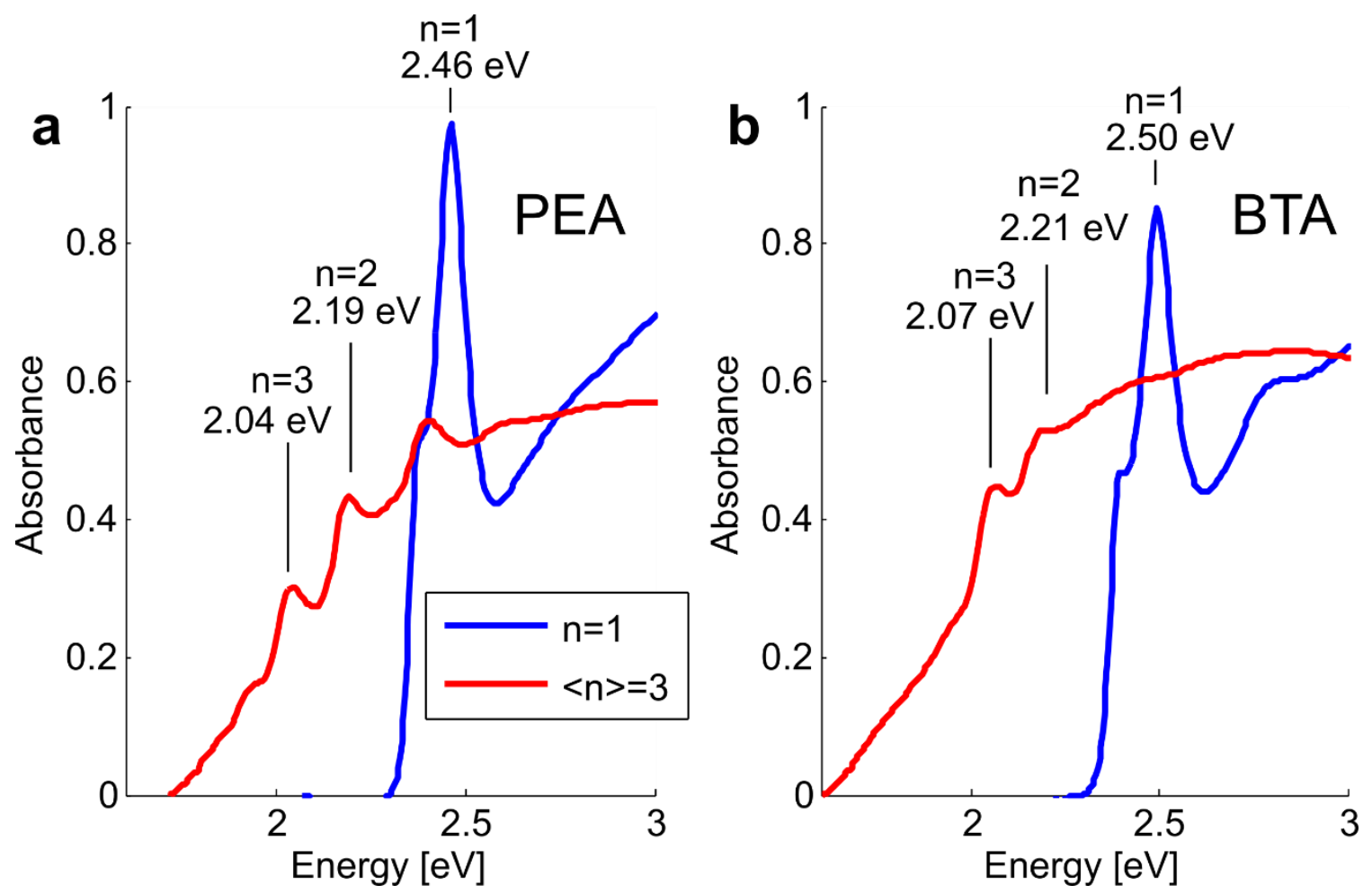

Figure S9. Absorption spectra of PEA and BTA RDP films. The exciton resonance energy for $\mathrm{n}=1,2$, and 3 is determined in $\mathrm{n}=1$ and $\mathrm{n}=3$ RDP films.
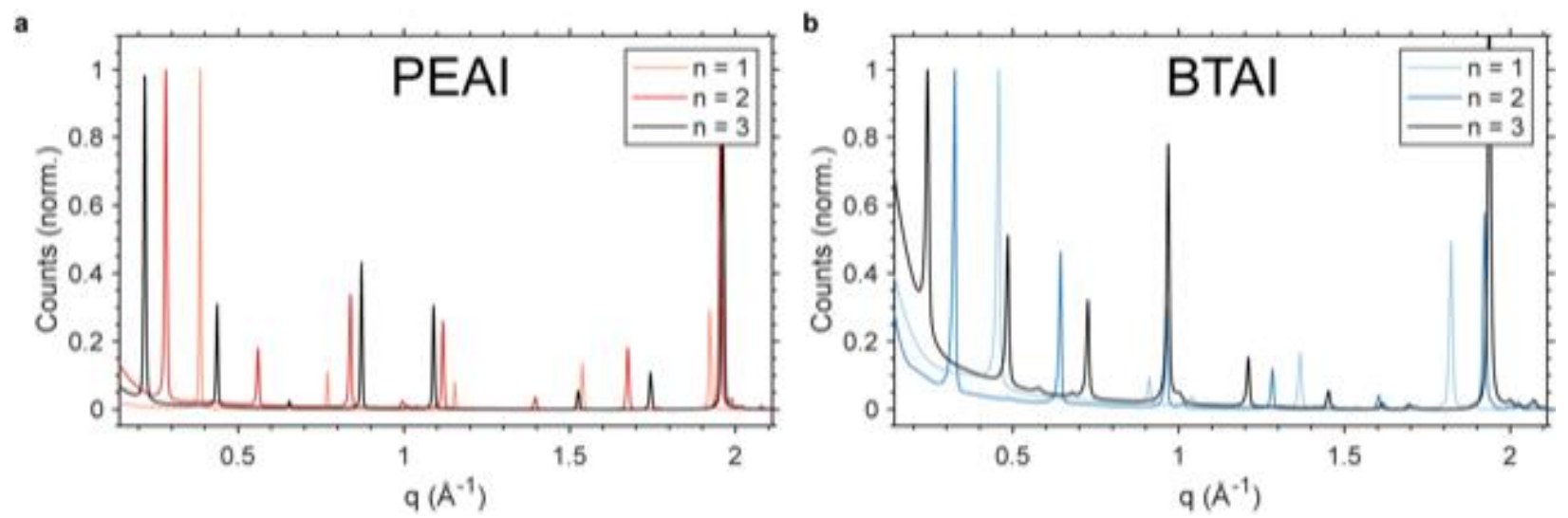

Figure S10. Powder X-ray Diffraction of the single crystals analyzed in Figure 1. As has been shown previously in the literature, single crystals exhibit a much higher phase purity than RDP films. 

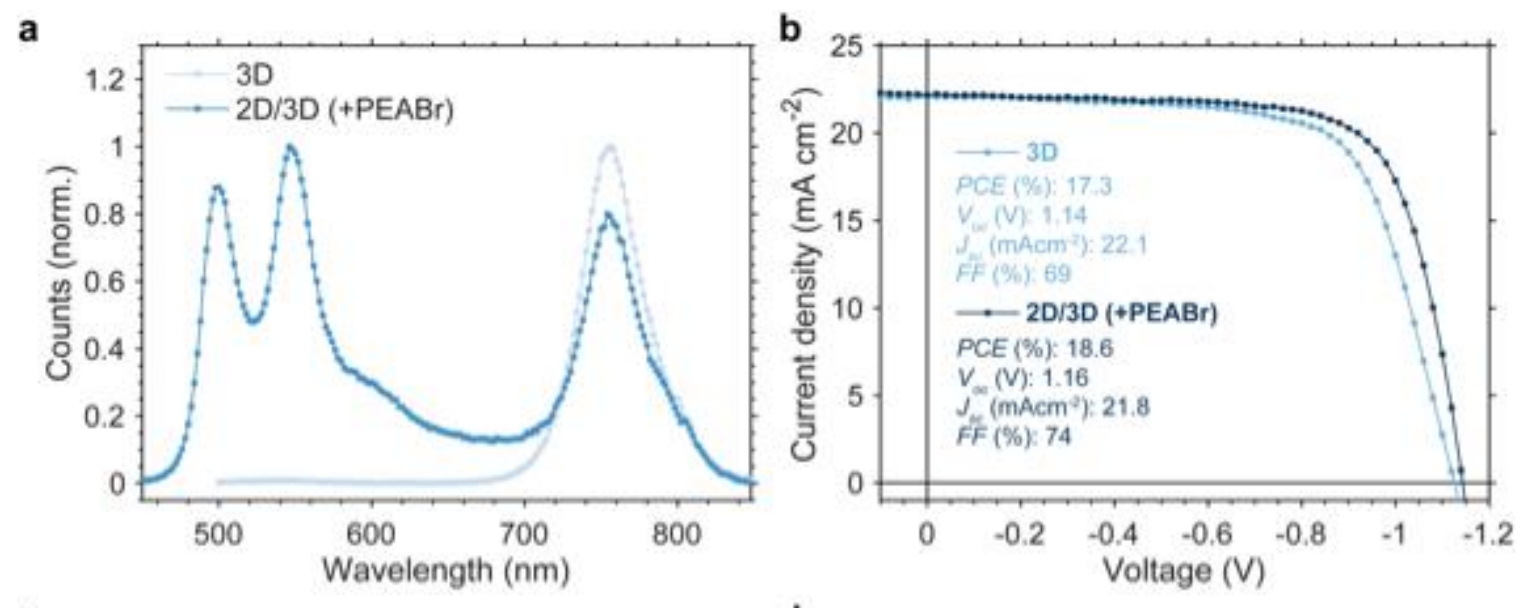

c

d

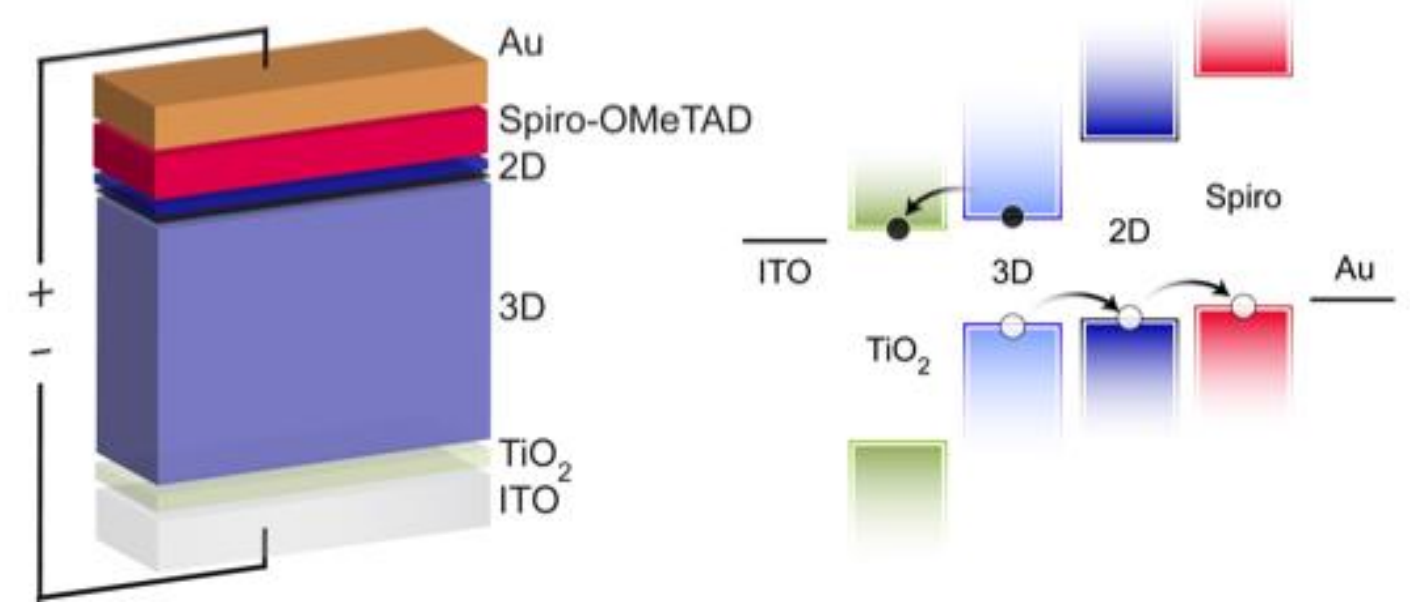

Figure S11. Thin layer of 2D perovskite improve photovoltaic metrics. (a)

Photoluminescence spectra for 3D-only and 2D/3D thin films. Films were excited using a laser diode with a wavelength of $374 \mathrm{~nm}$. 2D/3D films were synthesized by treating annealed 3D-only films with a solution of $2.5 \mathrm{mg} / \mathrm{mL}$ phenethylammonium bromide (PEABr) dissolved in isopropanol:chlorobenzene (in a 1:1 ratio), followed by subsequent annealing at $100^{\circ} \mathrm{C}$ for 10 minutes. The treatment was applied to make the $2 \mathrm{D} / 3 \mathrm{D}$ devices. (b) Current-voltage (J-V) curves for photovoltaic devices using 3D-only and 2D/3D active layers. (c) n-i-p device architecture comprised of ITO / TiO2 / 3D perovskite / 2D perovskite / Spiro-OMeTAD / Au and (d) schematic energy diagram for the various layers within the device. 


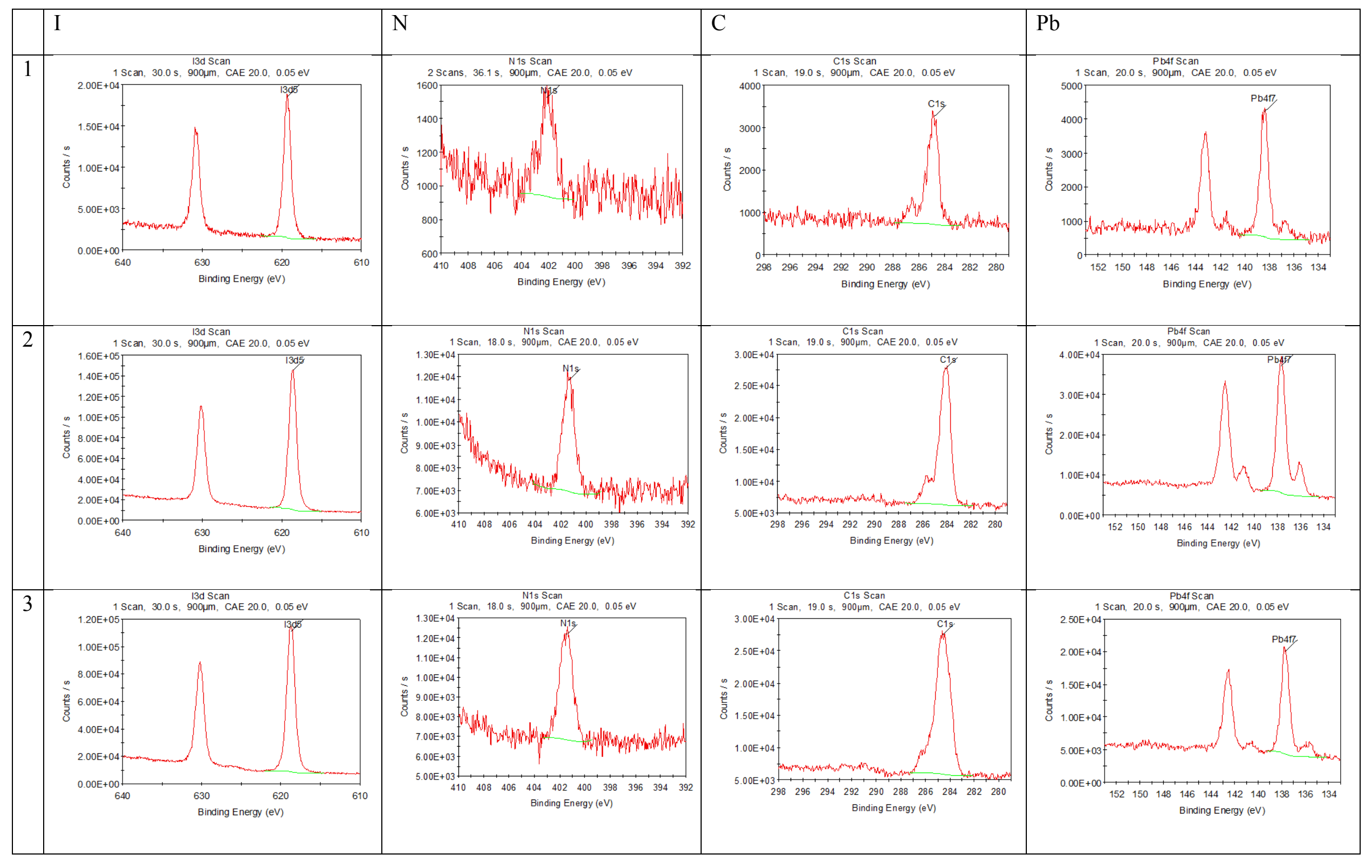




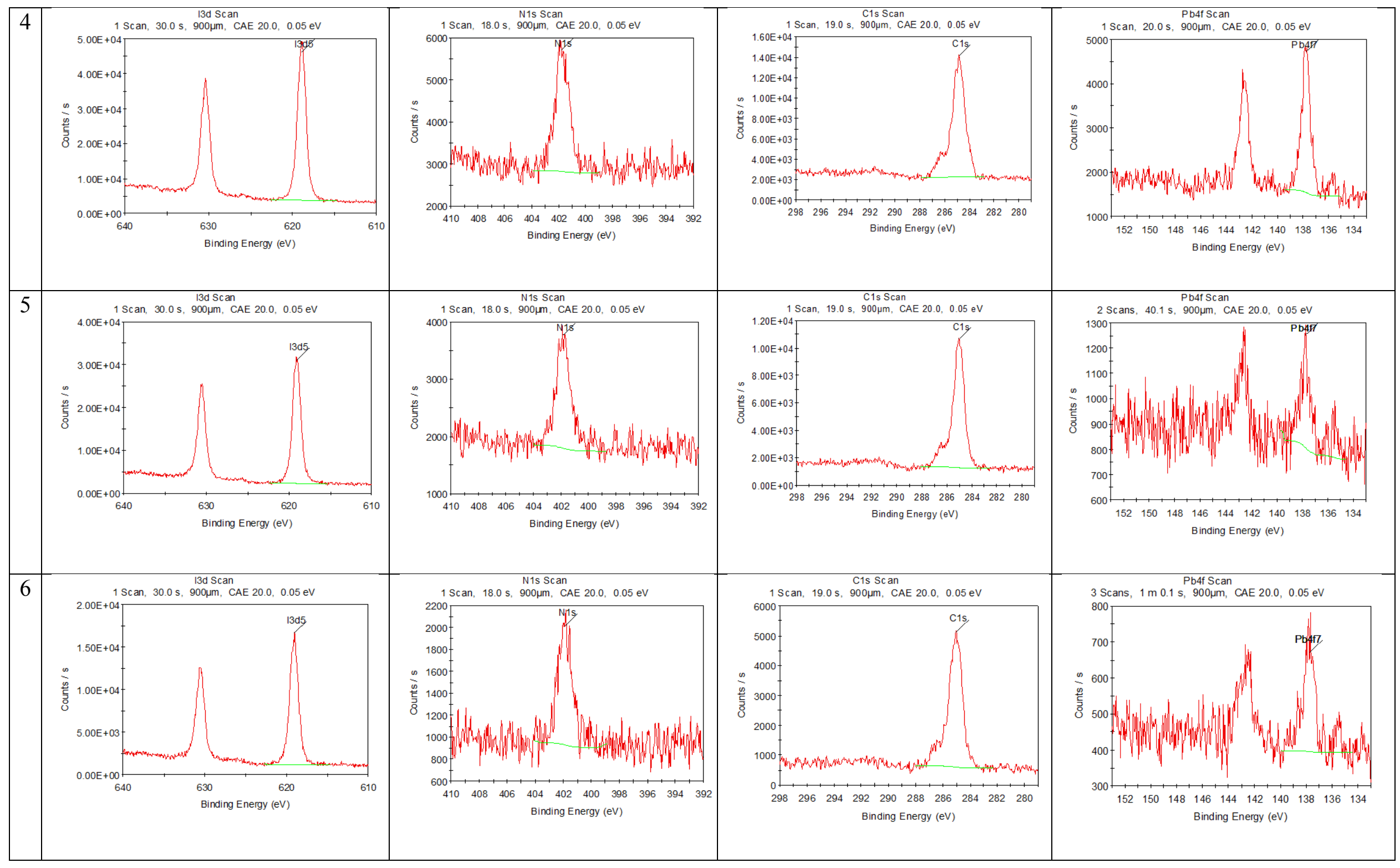




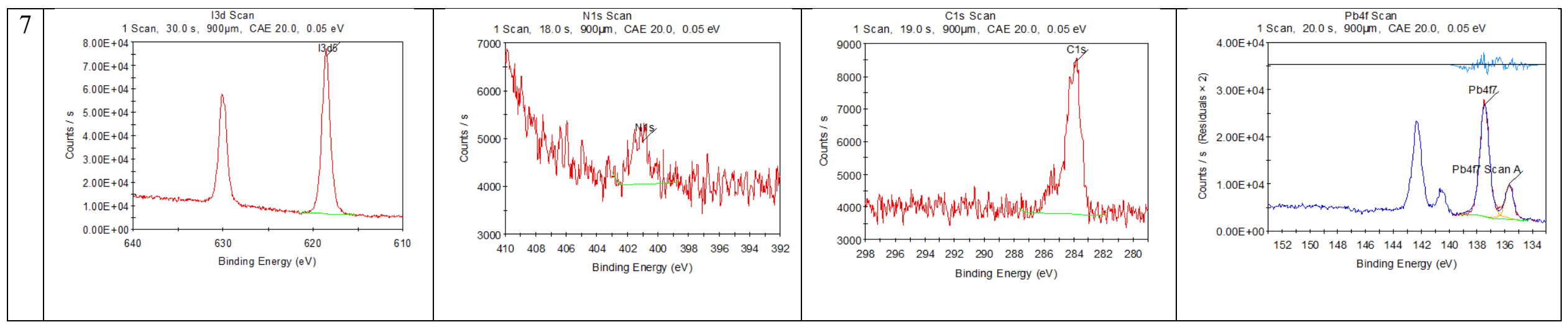

Figure S12. Raw XPS spectra of $\mathbf{n}=\mathbf{1}$ films pre- and post-treatments. The numbers denote the following: $1-\mathrm{control}, 2-2 \mathrm{mg} / \mathrm{mL} P E A I, 3-4 \mathrm{mg} / \mathrm{mL}$ PEAI, 4 - 6mg/mL PEAI, 5 - 8mg/mL PEAI, 6 - 10mg/mL PEAI, and 7 - maximum concentration of $\mathrm{PbI}_{2}$.

Table S1. Surface energies of PEAI $n=1$ phases with various surface terminations. Surface energies computed by DFT. The labels in brackets refer to the atomic simulation schematics on Figure $3 \mathrm{~d}$.

\section{No PEAI With PEAI vacancy Full PEAI-terminated}

\begin{tabular}{l|ccc}
\hline 001 Surface & $1.18 \mathrm{eV} / \mathrm{nm}^{2}$ (i) & $2.03 \mathrm{eV} / \mathrm{nm}^{2}$ (ii) & $1.02 \mathrm{eV} / \mathrm{nm}^{2}$ (iii) \\
100 Surface & $0.94 \mathrm{eV} / \mathrm{nm}^{2}$ (iv) & $0.99 \mathrm{eV} / \mathrm{nm}^{2}$ (v) & $1.30 \mathrm{eV} / \mathrm{nm}^{2}$ (vi)
\end{tabular}

